\title{
Regioselective iodination of hydroxylated aromatic ketones
}

\author{
Bhagwan R. Patil ${ }^{a}$, Sudhakar R. Bhusare ${ }^{\text {c* }}$, Rajendra P. Pawar ${ }^{a}$, \\ and Yeshwant B. Vibhute ${ }^{b_{*}}$ \\ ${ }^{a}$ Organic Chemistry Synthesis Lab. Dnyanopasak College, Parbhani-431401, India. \\ ${ }^{b}$ P. G. Department of Chemistry, Yeshwant Mahavidyalaya, Nanded-431 602, India. \\ ${ }^{c}$ Department of Chemistry, Shri Shivaji College, Parbhani-431401, India. \\ E-mail:bhusare71@yahoo.com
}

\begin{abstract}
A variety of ortho hydroxy substituted aromatic carbonyl compounds were regioselectively mono or diiodinated with iodine and iodic acid in excellent yields.
\end{abstract}

Keywords: Benzophenone, butyrophenone, iodination, molecular iodine, iodic acid

\section{Introduction}

Iodohydroxy aromatic ketones can be prepared by Fries rearrangement ${ }^{1}$ of iodinated phenyl aromatic esters, however, after the Fries rearrangement, steam distillation is required to separate the isomers which is a time consuming method and iodophenols are not easily available.

In recent years, direct iodination methods have been intensively developed using iodinium donating systems, such as iodine nitrogen dioxide, ${ }^{2}$ iodine-F-TEDA-[1-chloromethyl-4-fluoro1,4-diazoniabicyclo[2,2,2] octane-bis-(tetrafluoroborate)], ${ }^{3} \quad$ bis- $N$-iodosuccinimide, ${ }^{4}$ iodine-diiodine pentoxide, ${ }^{5}$ mercury(II)-oxide-iodine, ${ }^{6}$ iodine monochloride, ${ }^{7}$ bis(pyridine)iodonium(I) tetrafluoroborate $\mathrm{CF}_{3} \mathrm{SO}_{3} \mathrm{H},{ }^{8}$ NIS- $\mathrm{CF}_{3} \mathrm{SO}_{3} \mathrm{H},{ }^{9}$ iodine silver sulfate, ${ }^{10}$ iodine-mercury salts, ${ }^{11}$ $\mathrm{NaOCl}-\mathrm{NaI},{ }^{12}$ iodine/ $\mathrm{Na}_{2} \mathrm{~S}_{2} \mathrm{O}_{8}{ }^{13}$ and iodine- $\left(\mathrm{NH}_{4}\right)_{2} \mathrm{~S}_{2} \mathrm{O}_{8}-\mathrm{CuCl}_{2}-\mathrm{Ag}_{2} \mathrm{SO}_{4}{ }^{14}$ Iodination of resorcinol derivatives and hydroxy chromones has been carried out using iodine and sodium hydroxide, ${ }^{15}$ potassium iodide and potassium iodate ${ }^{16}$ in acetic acid medium. However, most of these methods involve hazardous or toxic reagents. 


\section{Result and Discussion}

We here report a practical and regioselective aromatic iodination. A combination of iodine and iodic acid has been found to be an excellent reagent for the efficient iodination of aromatic carbonyl compounds such as hydroxy butyrophenones and hydroxy benzophenones. These reactions are carried out at $35-40{ }^{\circ} \mathrm{C}$ using commercial $95 \%$ aqueous ethyl alcohol as a solvent.

A variety of ortho hydroxy substituted aromatic carbonyl compounds were selected for the iodination reaction using iodine and iodic acid. The iodination occurs regioselectively and the Ciodination took place at ortho or/and para positions. When the o-position was blocked with a substituent, then iodination took place at $p$-position and vice versa. The diiodination occurs if ortho and para positions are unsubstituted. Iodination does not occur in the side chain i.e. -CO$\mathrm{CH}_{2}-\mathrm{R}$ or $-\mathrm{CH}_{3}$; only nuclear iodination takes place. The iodination procedure is very simple; chemicals are not hazardous and can be weighed easily.

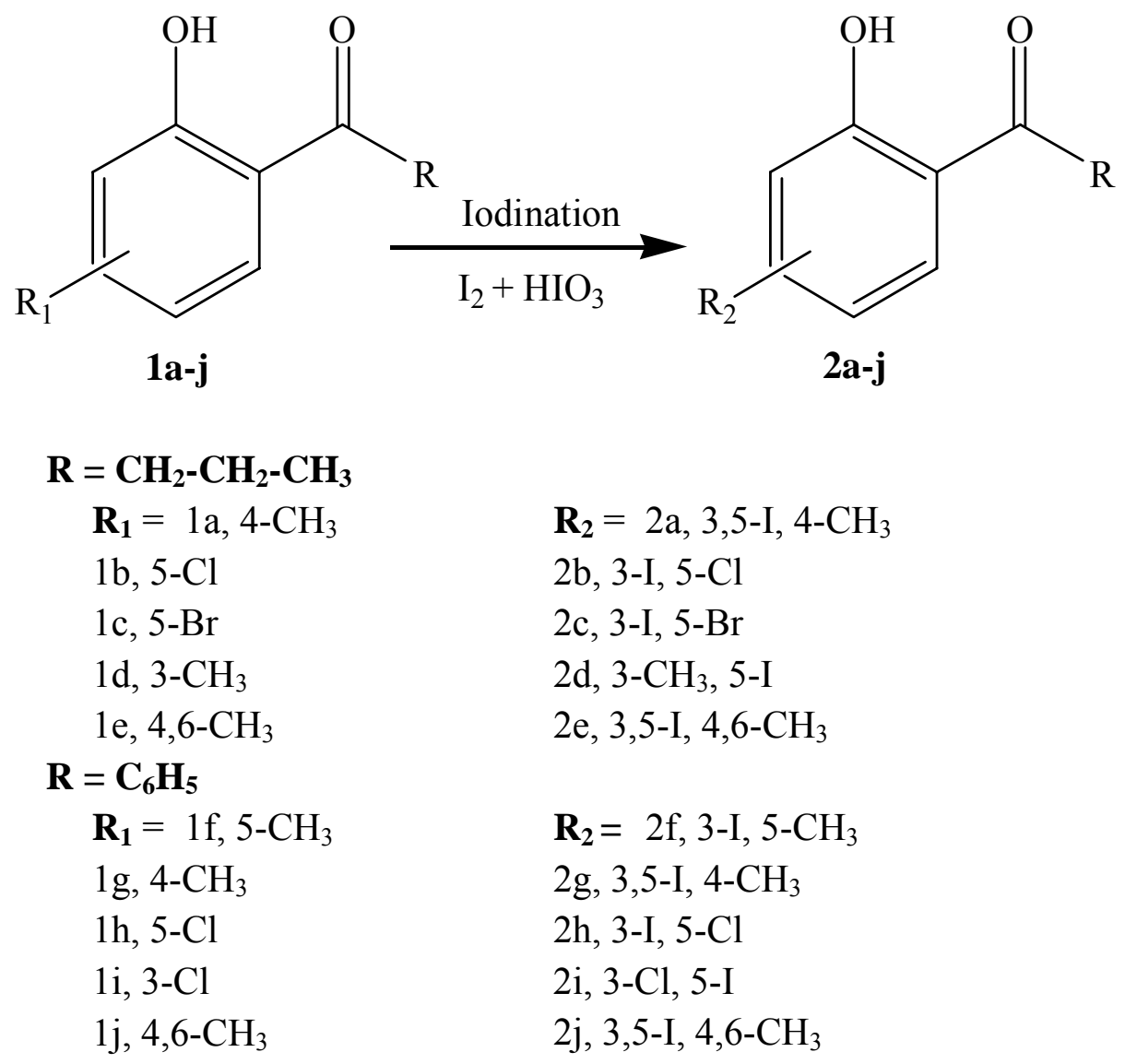

\section{Scheme 1}




\section{Experimental Section}

General Procedures. Melting points were determined in open capillary tubes and are uncorrected. The purity of the compounds has been checked by TLC. The IR spectra were recorded on FTIR Perkin-Elmer Spectrometer; ${ }^{1} \mathrm{H}$ NMR spectra were recorded on Gemini 300 $\mathrm{MHz}$ in $\mathrm{CDCl}_{3}$ as a solvent and TMS as an internal standard. Mass spectra are recorded on VG $7070 \mathrm{H}$ mass spectrometer.

General procedure for iodination of hydroxy aromatic ketones by iodine and iodic acid (2a2j). To a mixture of 2-hydroxy aryl ketones $(0.05 \mathrm{~mol})$ and iodine $(0.02 \mathrm{~mol})$ dissolved in ethanol $(30 \mathrm{~mL})$, iodic acid $(0.01 \mathrm{~mol})$ dissolved in water $(1 \mathrm{~mL})$ was added while stirring for 10 min. The reaction mixture was then stirred for $1.5 \mathrm{~h}$ at $35-40{ }^{0} \mathrm{C}$. The solid product separates out on dilution with water $(15-20 \mathrm{~mL})$. It was filtered, washed with saturated sodium thiosulphate solution to remove the excess of iodine, washed with cold water and crystallized from ethyl alcohol.

1-(2-Hydroxy-3, 5-diiodo-4-methyl-phenyl) butan-1-one (2a). Yield 81\%, mp $98{ }^{\circ} \mathrm{C}$; IR (cm ${ }^{-1}$ ) $1633(\mathrm{C}=\mathrm{O}), 1470(\mathrm{C}=\mathrm{C}), 1162(\mathrm{C}-\mathrm{O}) .{ }^{1} \mathrm{H} \mathrm{NMR}\left(\mathrm{CDCl}_{3}\right) \delta: 0.97$ (t, 3H, $\left.\mathrm{CH}_{2}-\mathrm{CH}_{2}-\mathrm{CH}_{3}\right), 1.72$ (m, $2 \mathrm{H}, \mathrm{CH}_{2}-\mathrm{CH}_{2}-\mathrm{CH}_{3}$ ), 2.86 (t, $\left.2 \mathrm{H}, \mathrm{CH}_{2}-\mathrm{CH}_{2}-\mathrm{CH}_{3}\right), 2.35$ (s, 3H, 4Ar- $\mathrm{CH}_{3}$ ), 8.10 (s, 1H, 6Ar$\mathrm{H}) ;{ }^{13} \mathrm{C}$ NMR $\delta 15.7,19.8,21.4,47.7,89.3,96.8,124.1,133.5,159.7,166.9,198.6$. M/z (relative intensity): $\mathrm{M}^{+} 430$ (20), 261 (100), 105 (22), 77 (24), 51 (12). Anal. calcd for $\mathrm{C}_{11} \mathrm{H}_{12} \mathrm{I}_{2} \mathrm{O}_{2}$ : C, 30.72; H, 2.81; I, 59.02. Found: C, 30.67; H, 2.77; I, 59.10.

1-(2-Hydroxy-3-iodo-5-chloro-phenyl) butan-1-one (2b). Yield $76 \%$, mp $163{ }^{\circ} \mathrm{C}$; IR (cm- ${ }^{1}$ )

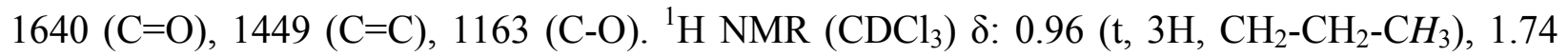
(m, 2H, $\left.\mathrm{CH}_{2}-\mathrm{CH}_{2}-\mathrm{CH}_{3}\right), 2.86$ (t, 2H, $\left.\mathrm{CH}_{2}-\mathrm{CH}_{2}-\mathrm{CH}_{3}\right), 7.75$ (s, 1H, 6Ar-H), 8.13 (s, 1H, 4Ar-H). $\mathrm{M} / \mathrm{z}$ (Relative intensity): $\mathrm{M}^{+} 324$ (38), 281 (100), 126 (21), 91 (17), 63 (10). Anal. calcd for $\mathrm{C}_{10} \mathrm{H}_{10} \mathrm{ClIO}_{2}$ : C, 37.01; H, 3.11; I, 39.10. Found: C, 37.06; H, 3.07; I, 39.18.

1-(2-Hydroxy-3-iodo-5-bromo-phenyl) butan-1-one (2c). Yield 78\%, mp $127{ }^{\circ} \mathrm{C}$; IR (cm- $\left.{ }^{1}\right)$ $1629(\mathrm{C}=\mathrm{O}), 1565(\mathrm{C}=\mathrm{C}), 1151(\mathrm{C}-\mathrm{O}) .{ }^{1} \mathrm{H} \mathrm{NMR}\left(\mathrm{CDCl}_{3}\right) \delta: 0.97$ (t, 3H, $\left.\mathrm{CH}_{2}-\mathrm{CH}_{2}-\mathrm{CH}_{3}\right), 1.72$ (m, 2H, $\left.\mathrm{CH}_{2}-\mathrm{CH}_{2}-\mathrm{CH}_{3}\right), 2.85$ (t, 2H, $\left.\mathrm{CH}_{2}-\mathrm{CH}_{2}-\mathrm{CH}_{3}\right), 7.8$ (s, 1H, 6Ar-H), 8.15 (s, 1H, 4Ar-H). $\mathrm{M} / \mathrm{z}$ (Relative intensity): $\mathrm{M}^{+} 368$ (32), 325 (100), 170 (28), 91 (13), 63 (08). Anal. calcd for $\mathrm{C}_{10} \mathrm{H}_{10} \mathrm{BrIO}_{2}$ : C, 32.55; H, 2.73; I, 34.39. Found: C, 32.64; H, 2.83; I, 34.46.

1-(2-Hydroxy-3-methyl-5-iodo-phenyl) butan-1-one (2d). Yield $\quad 80 \%$, mp $119{ }^{\circ} \mathrm{C}$; IR $\left(\mathrm{cm}^{-1}\right) 1637(\mathrm{C}=\mathrm{O}), 1580(\mathrm{C}=\mathrm{C}), 1158(\mathrm{C}-\mathrm{O}) .{ }^{1} \mathrm{H} \mathrm{NMR}\left(\mathrm{CDCl}_{3}\right)$ \&: $0.98\left(\mathrm{t}, 3 \mathrm{H}, \mathrm{CH}_{2}-\mathrm{CH}_{2}-\mathrm{CH}_{3}\right)$, $1.71\left(\mathrm{~m}, 2 \mathrm{H}, \mathrm{CH}_{2}-\mathrm{CH}_{2}-\mathrm{CH}_{3}\right), 2.85\left(\mathrm{t}, 2 \mathrm{H}, \mathrm{CH}_{2}-\mathrm{CH}_{2}-\mathrm{CH}_{3}\right), 2.33$ (s, 3H, 3Ar-CH $), 7.78$ (s, 1H, 4Ar-H), 8.15 (s, 1H, 6Ar-H). M/z (Relative intensity): $\mathrm{M}^{+} 304$ (32), 261 (100), 106 (14), 77 (21), 51 (10). Anal. calcd for $\mathrm{C}_{11} \mathrm{H}_{13} \mathrm{IO}_{2}$ : C, 43.44; H, 4.31; I, 41.73. Found: C, 43.57; H, 4.25; I, 41.70 .

1-(2-Hydroxy-3, 5-diiodo-4, 6-dimethyl-phenyl) butan-1-one (2e). Yield 75\%, mp $181^{\circ} \mathrm{C}$; IR $\left(\mathrm{cm}^{-1}\right): 1649(\mathrm{C}=\mathrm{O}), 1585(\mathrm{C}=\mathrm{C}), 1158(\mathrm{C}-\mathrm{O}) .{ }^{1} \mathrm{H} \mathrm{NMR}\left(\mathrm{CDCl}_{3}\right): \delta 0.97\left(\mathrm{t}, 3 \mathrm{H}, \mathrm{CH}_{2}-\mathrm{CH}_{2}-\mathrm{CH}_{3}\right)$, $1.77\left(\mathrm{~m}, 2 \mathrm{H}, \mathrm{CH}_{2}-\mathrm{CH}_{2}-\mathrm{CH}_{3}\right), 2.85$ (t, 2H, $\left.\mathrm{CH}_{2}-\mathrm{CH}_{2}-\mathrm{CH}_{3}\right), 2.35$ (s, 3H, 4Ar- $\left.\mathrm{CH}_{3}\right), 2.35$ (s, 3H, 
6Ar- $-\mathrm{CH}_{3}$ ). M/z (Relative intensity): $\mathrm{M}^{+} 444$ (40), 401 (100), 126 (25), 104 (18), 77 (23), 51 (08). Anal. calcd for $\mathrm{C}_{12} \mathrm{H}_{14} \mathrm{I}_{2} \mathrm{O}_{2}$ : C, 32.46; H, 3.18; I, 57.16. Found: C, 32.42; H, 3.21; I, 57.11 .

(2-Hydroxy-3-iodo-5-methyl phenyl) phenyl methanone (2f). Yield $82 \%, \mathrm{mp} 80^{\circ} \mathrm{C}$; IR $\left(\mathrm{cm}^{-1}\right)$ : $1627(\mathrm{C}=\mathrm{O}), 1598(\mathrm{C}=\mathrm{C}), 1188(\mathrm{C}-\mathrm{O}) .{ }^{1} \mathrm{H}$ NMR $\left(\mathrm{CDCl}_{3}\right) \delta: 2.23\left(\mathrm{~s}, 3 \mathrm{H}, \mathrm{Ar}_{-} \mathrm{CH}_{3}\right), 7.75-7.83(\mathrm{~m}$, $7 \mathrm{H}, \mathrm{Ar}-\mathrm{H}) ;{ }^{13} \mathrm{C}$ NMR $\delta 20.8,87.4,127.3,129.2,129.9,130.2,130.6,131.3,131.8,133.7,138.9$, 144.7, 167.8, 190.2. M/z (Relative intensity): $\mathrm{M}^{+} 337$ (38), 261 (100), 105 (58),77 (20), 51 (52). Anal. calcd for $\mathrm{C}_{14} \mathrm{H}_{11} \mathrm{IO}_{2}$ : C, 49.73; H, 3.28; I, 37.53. Found: C, 49.77; H, 3.31; I, 37.59 .

(2-Hydroxy-3,5-diiodo-4-methyl phenyl) phenyl methanone (2g). Yield $\quad 84 \%, \mathrm{mp} \quad 121^{\circ} \mathrm{C}$;

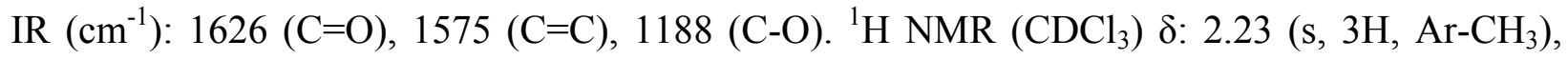
7.25-8.03 (m, 6H, Ar-H). M/z (Relative intensity): $\mathrm{M}^{+} 464$ (63), 338 (100), 261 (37), 105 (66), 77 (64), 51 (20). Anal. calcd for $\mathrm{C}_{14} \mathrm{H}_{10} \mathrm{I}_{2} \mathrm{O}_{2}$ : C, 36.24; H, 2.17; I, 54.70. Found: C, 36.25; H, 2.21; I, 54.74 .

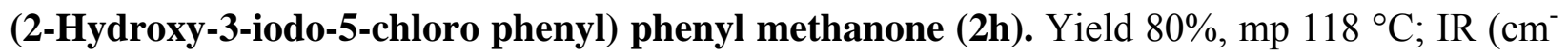

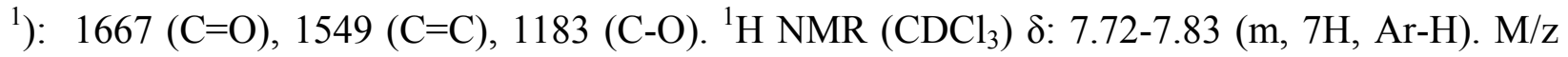
(Relative intensity): $\mathrm{M}^{+} 358$ (68), 281 (100), 105 (67), 77 (62), 51 (24). Anal. calcd for $\mathrm{C}_{13} \mathrm{H}_{8} \mathrm{ClIO}_{2}$ : C, 43.55; H, 2.25; I, 35.39. Found: C, 43.61; H, 2.27; I, 35.42.

(2-Hydroxy-3-chloro-5-iodo phenyl) phenyl methanone (2i). Yield 82\%, mp $172{ }^{\circ} \mathrm{C}$; IR (cm ${ }^{-}$ $\left.{ }^{1}\right): 1644(\mathrm{C}=\mathrm{O}), 1579(\mathrm{C}=\mathrm{C}), 1131(\mathrm{C}-\mathrm{O}) .{ }^{1} \mathrm{H}$ NMR $\left(\mathrm{CDCl}_{3}\right)$ 8: 7.47-7.82 (m, 7H, Ar-H). M/z (Relative intensity): $\mathrm{M}^{+} 358$ (24), 281 (100), 105 (64), 77 (62), 51 (18). Anal. calcd for $\mathrm{C}_{13} \mathrm{H}_{8} \mathrm{ClIO}_{2}$ : C, 43.55; H, 2.25; I, 35.39. Found: C, 43.64; H, 2.24; I, 35.44.

(2-Hydroxy-3,5-diiodo-4,6-dimethyl phenyl) phenyl methanone (2j). $\quad$ Yield $75 \%, \mathrm{mp}$ $133{ }^{\circ} \mathrm{C}$; IR $\left(\mathrm{cm}^{-1}\right): 1625(\mathrm{C}=\mathrm{O}), 1592(\mathrm{C}=\mathrm{C}), 1188(\mathrm{C}-\mathrm{O}) .{ }^{1} \mathrm{H}$ NMR $\left(\mathrm{CDCl}_{3}\right) \delta: 2.23$ (s, 3H, Ar$\left.\mathrm{CH}_{3}\right), 2.24$ (s, 3H, Ar-CH 3$), 7.21-7.86$ (m, 5H, Ar-H). M/z (Relative intensity): $\mathrm{M}^{+} 478$ (64), 401 (100), 121 (72), 106 (64),77 (50), 51 (20). Anal. calcd for $\mathrm{C}_{15} \mathrm{H}_{12} \mathrm{I}_{2} \mathrm{O}_{2}$ : C, 37.69; H, 2.53; I, 53.09. Found: C, 37.65; H, 2.58; I, 53.05.

\section{Acknowledgements}

The authors are thankful to the Principal, Yeshwant Mahavidyalaya, Nanded and Principal, Dnyanopasak Mahavidyalaya, Parbhani for providing laboratory facilities and Director of IICT Hyderabad for providing spectra's of samples. Authors are also thankful to UGC, New Delhi for sanctioning major research grant. 


\section{References}

1. Blatt, A. H. Org. React. 1942, 1, 342.

2. Noda, Y.; Kashima, M. Tetrahedron Lett. 1997, 38, 6225.

3. Zupan, M.; Iskra, J.; Stavber, S. Tetrahedron Lett. 1997, 38, 6305.

4. Carreno, M. C.; Ruano, J. L. G.; Sanz, G.; Toledo, M. A.; Urbano, A. Tetrahedron Lett. 1996, 37, 4081.

5. Brazdil, I. C.; Cuter, C. J. J. Org. Chem. 1996, 61, 9621.

6. Orito, K.; Hatakeyama, T.; Takeo, M.; Suginone, H.; Synthesis 1995, 1273.

7. Hubig, S. M.; Jung, W.; Kochi, J. K. J. Org. Chem. 1994, 59, 6233.

8. Barluenga, J.; Gonzalez, J. M.; Garcia-Martin, M. A.; Campos, P. J.; Asensia, G. J. Org. Chem. 1993, 58, 2058.

9. Olah, G. A.; Wang, D.; Sandford, G.; Prakash, G. K. S. J. Org. Chem. 1993, 58, 3194.

10. Sy, W. W. Tetrahedron Lett. 1993, 34, 6223.

11. Bachki, A.; Foubelo, F.; Yus, M. Tetrahedron Lett, 1994, 50, 5139.

12. Edgar, K. J.; Falling, S. N., J. Org. Chem., 1990, 55, 5287.

13. Elbs, K.; Jaroslawzee, A. J. Prakt. Chem., 1913, 88, 92.

14. Marko, D. M.; Belyoew. U. A. Khim, Referat, Zhur. 1941, 4, 49.

15. Hisao, J. Pharm. Soc. Jpn. 1943, 13, 63. (Chem. Abstr. 1950, 44, 72650)

16. Segalle, Monatsh. Chem., 1896, 17, 314. 